\title{
Human articular chondrocytes on macroporous gelatin microcarriers form structurally stable constructs with blood-derived biological glues in vitro
}

Sofia Pettersson, Jonas Wetterö, Pentti Tengvall and Gunnar Kratz

\author{
Linköping University Post Print
}

\section{Tweet}

N.B.: When citing this work, cite the original article.

Original Publication:

Sofia Pettersson, Jonas Wetterö, Pentti Tengvall and Gunnar Kratz, Human articular chondrocytes on macroporous gelatin microcarriers form structurally stable constructs with blood-derived biological glues in vitro, 2009, JOURNAL OF TISSUE ENGINEERING AND REGENERATIVE MEDICINE, (35), 6, 450-460.

http://dx.doi.org/10.1002/term.179

Copyright: Wiley

http://eu.wiley.com/WileyCDA/

Postprint available at: Linköping University Electronic Press

http://urn.kb.se/resolve?urn=urn:nbn:se:liu:diva-20597 


\section{Human articular chondrocytes on macroporous gelatin microcarriers form}

structurally stable constructs with blood-derived biological glues in vitro

Sofia Pettersson $^{1 *}$, Jonas Wetterö ${ }^{2}$, Pentti Tengvall ${ }^{3}$, Gunnar Kratz ${ }^{1,4}$

\section{Manuscript}

${ }^{1}$ Laboratory for Experimental Plastic Surgery, Dept. of Clinical and Experimental

Medicine, Linköping University, SE-581 85 Linköping, Sweden

${ }^{2}$ Rheumatology/AIR, Dept. of Clinical and Experimental Medicine, Linköping

University, SE-581 85 Linköping, Sweden

${ }^{3}$ Institute of Clinical Sciences, Dept. of Biomaterials, The Sahlgrenska Academy at University of Gothenburg, Gothenburg, Sweden

${ }^{4}$ Dept. of Hand-, Plastic-, and Burn Surgery, University Hospital of Linköping, SE58185 Linköping, Sweden

Work performed at the Laboratory for Experimental Plastic Surgery, Dept. of Clinical and Experimental Medicine, Linköping University, Linköping, Sweden

\section{*Correspondence to:}

Sofia Pettersson

E-mail: sofia.pettersson@liu.se

Phone: +4613227337

Fax: +4613127465

Running Head: Chondrocyte-seeded microcarriers in blood-derived glues

Key Words: cartilage; chondrocyte; microcarrier; tissue engineering; fibrin glue; blood plasma 
ABSTRACT

Biodegradable macroporous gelatin microcarriers fixed with blood-derived biodegradable glue is proposed as a delivery system for human autologous chondrocytes. Cell-seeded microcarriers were embedded in four biological glues recalcified citrated whole blood, recalcified citrated plasma with or without platelets, and a commercially available fibrin glue - and cultured in an in vitro model under static conditions for 16 weeks. No differences could be verified between the commercial fibrin glue and the blood-derived alternatives. Five further experiments were conducted with recalcified citrated platelet rich plasma alone as microcarrier sealant, using two different in vitro culture models and chondrocytes from three additional donors. The microcarriers were found chondrocyte adhesion and expansion, as well as extracellular matrix synthesis. Matrix formation occurred predominantly at sample surfaces under the static conditions. The presence of microcarriers proved essential for the glues to support the structural takeover of extracellular matrix proteins produced by the embedded chondrocytes, as exclusion of the microcarriers resulted in unstable structures that dissolved before matrix formation could occur. Immunohistochemical analysis revealed presence of SOX-9 and S-100 positive chondrocytes as well as production of aggrecan and collagen type I, but not of the cartilage-specific collagen type II. These results imply that blood-derived glues are indeed potentially applicable for encapsulation of chondrocyte-seeded microcarriers. However, the static in vitro models used in this study proved incapable of supporting cartilage formation throughout the engineered constructs. 


\section{INTRODUCTION}

Approaches towards tissue engineered cartilage combine chondrocytes with a biomaterial scaffold that is able to support adhesion of the anchorage dependent cells as well as formation of extracellular matrix (ECM). Microcarrier-based cell culture was originally introduced as a means to obtain large surface areas for anchorage-dependant cells [van Wezel, 1967]. It has since become clear that these scaffolds have potential beyond cell culture and the use of microcarriers as transplantation vehicles has been suggested previously by us as well as others [Demetriou et al., 1988, Voigt et al., 1999, Overstreet et al., 2003, Chun et al., 2004, Mercier et al., 2005, Huss et al., 2007]. In recent years, a wide range of microcarriers with different material properties and characteristics has been developed for tissue engineering of bone and cartilage [Malda and Frondoza, 2006]. The choice of microcarrier material and characteristics can indeed have a significant effect on the outcome [Frondoza et al., 1996, Kong et al., 1999, Malda et al., 2003b, Young et al., 2005]. Porous microcarriers further enhance the matrix culture area and interconnected pores enable migration of cells as well as cell-cell signaling within the microcarriers, facilitating a homogenous distribution of anchored cells and cell signals throughout the scaffold. Gelatin is a natural polymer derived from collagen that has been widely used as tissue engineering and delivery matrices due to it's biodegradable and biocompatible properties [Young et al., 2005]. Macroporous CultiSpher gelatin microcarriers have previously been shown to support cell adhesion and growth of several human and animal cell types [Ohlson et al., 1994, Werner et al., 2000, Malda et al., 2003a, Liu et al., 2004, Melero-Martin et al., 2006, Fernandes et al., 2007, Huss et al., 2007]. The microcarriers are easily injectable and this feature enables an efficient way to fill the wound site with a liquid, yet three-dimensional scaffold with well distributed cells. 
In order to make this approach useful, the cell-seeded microcarriers may need to be secured in a three-dimensional state until matrix production and tissue integration occurs. Commercial fibrin glues present an attractive off-the-shelf alternative, and have been investigated for cartilage engineering purposes, but concerns exist regarding their animal origin as well as their limitations when used as matrices for cells [Homminga et al., 1993, Brittberg et al., 1997, Meinhart et al., 1999, Silverman et al., 1999]. Bloodderived, autologous alternatives would however eliminate such concerns. With the clinical aim of combining a matrix that can act as a cell expansion vehicle as well as a transplantation matrix with an autologous adhesive, we hypothesize that a combination of macroporous gelatin microcarriers and a blood-derived biological glue can be used as a delivery system for cultured human chondrocytes. In the present study, we therefore performed an initial long-term study with a total of three blood-derived biological glues and one commercially available fibrin glue in a novel composite system with chondrocyte-seeded microcarriers. Subsequent experiments were also performed to supplement the data regarding the suitability of blood-derived alternative for microcarrier encapsulation. For this purpose, we also investigated two basic in vitro models for preliminary assessment of chondrocyte proliferation and ECM formation under static conditions. The aims of this study were thus to evaluate these models for cartilage formation in vitro in the absence of chondrogenic growth factors and to investigate the suitability of blood-derived biological glues in a gelatin microcarrier system.

\section{MATERIALS AND METHODS}

\subsection{Culture conditions}

Unless otherwise indicated all chemicals were obtained from Gibco ${ }^{\circledR}$ (Invitrogen Corp., 
Carlsbad, CA, USA). Two cell culture media were used during the course of this study. Basal medium, BM, refers to Dulbecco's modified Eagle's medium, DMEM, supplemented with $10 \%$ heat inactivated $\left(45 \mathrm{~min}\right.$ at $\left.57^{\circ} \mathrm{C}\right)$ newborn calf serum $(\mathrm{NCS})$, penicillin $(50 \mathrm{U} / \mathrm{mL})$ and streptomycin $(50 \mathrm{mg} / \mathrm{mL})$. This medium was later advanced to a chondrocyte growth medium, CM, that refers to basal medium with $10 \mathrm{mM}$ HEPES, $0.1 \mathrm{mM}$ non-essential amino acids, $0.4 \mathrm{mM}$ L-proline and $50 \mathrm{mg} / \mathrm{L} \mathrm{L}$-ascorbic acid. Cells and cartilage constructs were kept at $37^{\circ} \mathrm{C}, 5.0 \% \mathrm{CO}_{2}$ and $95 \%$ humidity for all cultures and medium was changed 3 times per week.

\subsection{Microcarriers}

Macroporous CultiSpher-GL gelatin microcarriers, manufactured from pharmaceutical grade porcine gelatin, were kindly supplied by the manufacturer (Percell Biolytica AB, Åstorp, Sweden). These microcarriers have a particle diameter of 166-370 $\mu \mathrm{m}$ and a mean internal pore size of $30 \mu \mathrm{m}$ when rehydrated in phosphate buffered saline (PBS). According to the manufacturer's recommendations, the microcarriers were rehydrated in $50 \mathrm{~mL} \mathrm{Ca}^{2+}$ - and $\mathrm{Mg}^{2+}$-free PBS (pH 7.4, Medicago AB, Uppsala, Sweden) per gram (dry weight) microcarrier for a minimum of one hour and sterilized by autoclaving $\left(121^{\circ} \mathrm{C}, 20 \mathrm{~min}, 80^{\circ} \mathrm{C}\right.$ cooling temperature, $\left.2.8 \mathrm{bar}\right)$. The sterilized carriers were washed twice in PBS, twice in cell culture medium, and stored at $4^{\circ} \mathrm{C}$ until further use. Prior to cell seeding, the microcarriers were pre-warmed with cell culture medium to $37^{\circ} \mathrm{C}$ in $250 \mathrm{~mL}$ spinner flasks (Techne, Staffordshire, U.K.).

\subsection{Chondrocyte isolation and seeding}

Human articular chondrocytes were isolated from four different donors following total knee arthroplasties. In accordance with national ethical regulations, cells were obtained from surgical waste tissue without knowledge of the donor identities. In short, $1-\mathrm{mm}^{3}$ 
pieces of articular cartilage were enzymatically digested overnight in a mixture of Dulbecco's modified Eagle's medium (DMEM), 2\% fetal calf serum (FCS), collagenase type II $(350 \mathrm{U} / \mathrm{mL})$ and dispase $(2.5 \mathrm{U} / \mathrm{mL})$. The resulting cell suspension was washed with cell culture medium and centrifuged at $293 \mathrm{~g}$ for $5 \mathrm{~min}$, resuspended and seeded onto $75 \mathrm{~cm}^{2}$ polystyrene cell culture flasks (BD, Franklin Lakes, NJ, USA) or directly onto gelatin microcarriers. Culture flask cells were passaged by washing the polystyrene flasks with $0.02 \%$ ethylene diamine tetra-acetic acid (EDTA) (ICN Biomedicals, Aurora, OH, USA) prior to enzymatic detachment with trypsin/EDTA $(0.125 \% / 0.01 \%)$ at $37^{\circ} \mathrm{C}$ for approximately $20 \mathrm{~min}$. Enzymatically detached chondrocytes (P0-P4) were washed in cell culture medium, centrifuged at $293 \mathrm{~g}$ for 5 min, resuspended, and counted in a Bürker chamber or using the ViaCount Assay with a Guava PCA counter (Guava Technologies, Hayward, CA, USA). Cells were added to the spinner flasks at cell concentrations ranging from $1 \times 10^{6}$ to $37 \times 10^{6}$ cells per $0.1 \mathrm{~g}$ microcarrier (dry weight) and $100 \mathrm{~mL}$ culture medium volume, and expanded on the microcarriers for 2-47 days before further use. The suspension was stirred intermittently (Techne MCS-104S, 20-35 rpm, 5 min stirring, 45-55 min non-stirring) for the initial 24-48 hours, and then stirred continuously.

\subsection{Cell viability and density}

Cell viability on CultiSpher-GL was evaluated with a 3-(4, 5-dimethylthiazol-2-yl)-2, 5diphenyltetrazolium bromide (MTT, Sigma-Aldrich Chemie GmbH, Steinheim, Germany) assay. In short, 0.4-mL samples of evenly distributed CultiSpher-GL suspensions were mixed with $40 \mathrm{~mL}$ MTT ( $5 \mathrm{mg} / \mathrm{mL}$ in PBS) each, and incubated for $45 \mathrm{~min}$ at $37^{\circ} \mathrm{C}$. Staining was evaluated using an Olympus IX51 inverted light microscope (x10/NA 0.25) and images were acquired with a high-resolution digital Olympus DP70 CCD camera and DP Controller software (version 1.1.1.65, Olympus 
Optical Co. Ltd, Tokyo, Japan). To monitor cell density, evenly distributed $0.5-\mathrm{mL}$ samples were collected from the stirrer flasks. After sedimentation of the microcarriers, the supernatant was discarded and the microcarriers were dissolved in trypsin at room temperature for approximately 20-30 minutes until the microcarriers had dissolved. The cell suspensions were thoroughly agitated and cells counted as previously stated.

\subsection{Biological glues}

Whole blood was drawn on the day of use from a non-medicated apparently healthy male donor to BD Vacutainer ${ }^{\circledR}$ collection tubes (BD Diagnostics, Plymouth, UK) containing $0.129 \mathrm{M}$ citrate. The tubes were stored at room temperature and agitated repeatedly. Platelet rich plasma was prepared by centrifugation of the citrated whole blood for $20 \mathrm{~min}$ at $220 \mathrm{~g}$. Platelet poor plasma was prepared by centrifugation of platelet rich plasma for $20 \mathrm{~min}$ at $480 \mathrm{~g}$ and additional centrifugation of the upper phase for another $10 \mathrm{~min}$ at $2000 \mathrm{~g}$. The resulting plasma fractions were collected to plastic tubes and stored briefly at room temperature. Clinical grade Tisseel ${ }^{\circledR}$ (Baxter International Inc., Deerfield, IL, USA) was thawed and the thrombin component diluted to $5 \mathrm{IU} / \mathrm{mL}$ in a $20 \mathrm{mM} \mathrm{CaCl}_{2}$ solution [Goessl and Redl, 2005]. [Platelet count should be adressed]

\subsection{In vitro model - cell culture inserts}

Cell-seeded microcarriers were collected from the spinner flasks, and excess culture medium removed and discarded. The microcarriers were mixed thoroughly with biological glue in a 1:1 volume ratio, and immediately distributed in prehydrated cell culture inserts. Unless otherwise stated, the coagulation process was initiated by adding $\mathrm{CaCl}_{2}$ to a final concentration of approximately $20 \mathrm{mM}$ for all samples. Samples were allowed to clot for $20 \mathrm{~min}$ at $37^{\circ} \mathrm{C}$ before adding cell culture medium to the well and 
insert.

\subsection{In vitro model - pellet culture}

Cell-seeded microcarriers were collected from the spinner flasks as previously described and divided into equal fractions in $5 \mathrm{~mL}$ polypropylene tubes (Sarstedt AG \& Co, Nümbrecht, Germany), resulting in approximately $8 \mathrm{mg}$ microcarriers per pellet. Excess culture medium was removed and 100-150 mL platelet rich plasma, prepared as described above, was added to each tube. The coagulation process was initiated by adding $\mathrm{CaCl}_{2}$ to a final concentration of approximately $20 \mathrm{mM}$ for all samples. Samples were allowed to clot for $20 \mathrm{~min}$ at $37^{\circ} \mathrm{C}$ before adding cell culture medium to the tubes.

\subsection{Initial evaluation of biological glues}

The biological glues were evaluated in four groups: A - Tisseel $\AA, B$ - platelet poor plasma, C - platelet rich plasma, and D - whole blood; using the cell culture insert model. Each microcarrier/glue mixture was distributed in $100 \mathrm{~mL}$ aliquots to prehydrated Collagen Type I coated 24-well Biocoat ${ }^{\circledR}$ cell culture inserts $(0.45 \mu \mathrm{m}$, Becton Dickinson Labware, Bedford, MA, USA) as described previously, resulting in approximately $2 \mathrm{~mm}$ high puck-shaped cylinders. For Tisseel ${ }^{\circledR}$, the thrombin was added to the microcarrier suspension immediately before distribution to cell culture inserts as described above. For each group, a set of microcarrier-free controls was prepared, containing detached monolayer chondrocytes $\left(5.90 \pm 1.7 \times 10^{5}\right.$ cells per cartilage sample) and biological glue only. For Tisseel $®$ controls, $150 \mathrm{~mL}$ Tissucol ${ }^{\circledR}$ was mixed with $300 \mathrm{~mL}$ concentrated cell suspension, mixed thoroughly and distributed into four inserts. $50 \mathrm{~mL}$ thrombin was subsequently added to each well. For blood-derived biological glues, the coagulation process was initiated by adding $\mathrm{CaCl}_{2}$ to a final concentration of approximately $20 \mathrm{mM}$ for all samples, including controls. After 4, 8, 
12 and 16 weeks, triplicate microcarrier samples and one control sample were taken for each group.

\subsection{Gelatin microcarriers and platelet rich plasma}

Five additional experiments have been completed using platelet rich plasma, prepared as described above, as biological glue. An overview of the included experiments with corresponding protocol adjustments, concerning cell culture media, cell passage number, in vitro model adjustments and sampling time points, can be found in Table 1. [Timeline with respect to Table 1 and PRP origin]

\subsection{Sampling}

Following each time point (see Table 1 for details), samples were fixed in $4 \%$ neutral buffered formaldehyde for 24 hours, washed, dehydrated in an ethanol-xylene series and embedded in Histowax (Histolab products AB, Gothenburg, Sweden). Seven-mm sections were prepared with a microtome (Leica Microsystems GmbH, Wetzlar, Germany), and mounted on Superfrost ${ }^{\circledR}$ Plus slides (Menzel GmbH \& Co KG, Braunschweig, Germany). Sections were deparaffinized and rehydrated in an ethanol series prior to staining.

\subsection{Histological and immunohistochemical staining}

For morphological assessment, sections were stained with Mayer's hematoxylin/eosin according to standard histological protocols. Sections were stained for proteoglycan content with $1 \%$ Alcian Blue (w/v in $0.1 \mathrm{M} \mathrm{HCl}$ ) followed by a $0.5 \mathrm{nM} 4$ '-6-Diamidino2-phenylindole (DAPI, Sigma-Aldrich) counterstain. For immunohistochemical analysis of aggrecan, sections were incubated in $0.1 \mathrm{M}$ glycine (Merck KgaA, Darmstadt, Germany) in PBS for $10 \mathrm{~min}$ to reduce autofluorescence. Antigen retrieval was performed with $0.1 \mathrm{U} / \mathrm{mL}$ Chondroitinase $\mathrm{ABC}$ (Sigma-Aldrich) in $50 \mathrm{mM}$ Tris-HCl 
buffer containing $60 \mathrm{mM}$ sodium acetate (Merck) at $\mathrm{pH} 8$ for $45 \mathrm{~min}$ at $37^{\circ} \mathrm{C}$. Nonspecific binding was blocked with bovine serum albumin (BSA, Sigma) prior to incubation with a mouse monoclonal aggrecan antibody (clone 6-B-4, Abcam plc, Cambridge, UK) diluted in PBS (1:50) for one hour, with negative controls incubated in PBS only. Sections were subsequently incubated with an Alexa Fluor ${ }^{\circledR} 488$ goat antimouse IgG antibody (Molecular Probes, Invitrogen) for one hour, and mounted with ProLong Gold antifade reagent containing DAPI (Molecular Probes). Sections were examined with an Olympus BX41 microscope, using ultraviolet and blue filters (MWU2 and MWIB2, Olympus). All images were acquired with a high-resolution digital Olympus DP70 CCD camera and DP Controller software.

\subsection{Image processing}

All image adjustments were made for the sole purpose of enhancing printing clarity, using Adobe Photoshop 6.0 (Adobe Systems Inc., San Jose, CA, USA). For montages of stained sections, identical adjustments were made for all equivalent images. Additional images were taken with a Sony DSC-W5 digital camera (Sony Corporation, Tokyo, Japan).

\section{RESULTS}

Viable primary human chondrocytes were found adhered to microcarriers in all samples tested prior to microcarrier encapsulation. The cells were homogeneously distributed among the microcarriers as well as on each individual microcarrier surface (Fig. 1 a). [Average number of cells..]

\subsection{Initial evaluation of blood-derived glues}

The microcarrier-free clots had dissolved completely in four weeks, with the exception 
of group A (Tisseel $\left.{ }^{\circledR}\right)$ where one solid clot was obtained at this time point. After eight weeks, no clots remained in the absence of supportive microcarriers. In contrast, cartilage samples containing cell-seeded microcarriers remained intact throughout the experiment. These allowed for convenient handling once removed from the inserts (Fig. $1 \mathrm{c})$.

Histological evaluation after four weeks showed that the cell density as well as the extracellular matrix (ECM) formation was concentrated to sample surfaces. Consequently, the amount of ECM, as evaluated by stained sections from later time points, also increased primarily at the sample perimeters. In the inner parts of the samples, cell densities decreased, and as a result, modest ECM formation occurred below the sample surfaces. The only remaining microcarrier-free sample at four weeks, from group A, displayed some cell accumulation at the surface. The cells residing within the sample could be found in lacunae-like cavities. There was however little or no evidence of cartilage matrix production (data not shown). In contrast, positive staining for sulphated proteoglycans and cell nuclei was observed for all groups containing pre-seeded microcarriers at all time points, while positive staining for aggrecan, the key proteoglycan in cartilage, could be found for all groups at the two last time points. The positive stain for matrix production corresponded well with the presence of cell nuclei. At the end of the 16-week culture period, no considerable differences, concerning cell density, capsule thickness or stain intensity, could be verified between the commercially available fibrin glue and the blood-derived glues. Consequently, positive staining was found in all experimental groups for sulphated proteoglycans (Fig. 2 a-d) and aggrecan (Fig. 2 e-h) after 16 weeks of culture. 


\subsection{Gelatin microcarriers and platelet rich plasma}

Subsequent experiments with encapsulation of chondrocyte-seeded microcarriers in platelet rich plasma revealed analogous results. With time, cell densities increased at the sample perimeters and decreased in the central parts of the engineered constructs, as illustrated by DAPI-stained nuclei (Fig. 3 a-c). As a result of this gradual cell accumulation toward the surfaces, ECM formation occurred mainly in a capsule surrounding the samples, as indicated by hematoxylin/eosin and alcian blue staining, while modest synthesis occurred below the capsule (Fig. 3 g-i). The sample diameters decreased with time under the static conditions, as indicated by a decrease in sample size during the in vitro culture (Fig. $1 \mathrm{~b}$ ) as well as in section diameters following staining (Fig. 3 a-i), arguably as a result of the lack of ECM formation in the center following the dramatic decrease in cellularity. In contrast, the cell densities and ECM quantities at the sample perimeters increased with time, as indicated by histological staining methods (Fig. 3, left to right). This result strongly suggests an active ECM synthesis of proliferative cells throughout the culture period. Also, a gradual increase in capsule thicknesses and cell densities has been noted during the course of this study between the included experiments (Fig. 4 top to bottom).

Immunohistochemical analysis of the ECM after 12 weeks of culture revealed no nonspecific labeling with the secondary antibody in negative controls. Cell-free microcarriers did not show any non-specific labeling with the primary antibodies with the exception of the anti-collagen type I antibody where some positive staining was detected, probably due to the origin of the gelatin. Principally, positive staining corresponded well with the overall morphology (Fig. 4 a, g, m) as well as the presence of cell nuclei and/or cytoplasm residues. The analysis of ECM-proteins confirmed positive staining for aggrecan for all samples after 12 weeks of culture (Fig. 4 b, h, n). 
There was no positive staining for the cartilage-specific collagen type II in any of the included experiments (Fig. 4 c, i, o), though staining for fibrocartilaginous collagen type I was detected in all samples (Fig. 4 d, j, p). On a cellular level, chondrocytes showed positive staining for S-100, a marker of the chondrocyte phenotype, after 12 weeks of culture (Fig. 4 e, k, q). The chondrogenic transcription factor SOX-9 was also detected in all samples (Fig. 4 f, 1, r). The anti-SOX-9 antibody showed no non-specific labeling with intact microcarriers, but associated with partially degraded gelatin microcarriers (Fig. 4 r).

\subsection{In vitro models}

Overall, the results from the two different in vitro models (Fig. 1 b) have been analogous, with one exception. For samples formed in cell culture inserts, a morphological difference between sample surfaces began to show during the later stages, manifested by their orientation in the cell culture inserts (data not shown). At the surface facing up towards the cell culture media interface, cells and the synthesized ECM were organized in a stratified, multi-layered manner, while the surface facing the porous membrane of the cell culture inserts, displayed a more random pattern of cell and ECM distribution. In contrast, samples that were formed in polypropylene tubes displayed a stratified multi-layer structure around the entire circumference of the constructs, although the capsule thickness varied around individual samples (illustrated in Fig. 3 f). For the experiments using exclusively microcarrier-expanded cells from the same donor (Experiments V and VI, Table 1), the microcarriers had started to degrade after 8 weeks (Fig. $3 \mathrm{~h}$ ) and this degradation was more pronounced after 12 weeks (Fig. 3 i). From an in vitro model point of view, the degradation was particularly noticeable for the pellet in vitro model (Experiment VI, Fig. 3 i). 


\section{DISCUSSION}

In the present study we have investigated the possibility to use biodegradable macroporous microcarriers as a matrix in human cartilage tissue engineering, by a series of static in vitro experiments. In addition, four different biological glues were initially evaluated with respect to their ability to facilitate microcarrier encapsulation during early cartilage tissue formation. To our knowledge this is the first study where this particular type of microcarrier has been investigated as a scaffold for human articular chondrocytes in combination with biological glues for long-term in vitro cultures. To enable these studies, two in vitro models were evaluated (Fig. 1 b). For this preliminary evaluation of the complex system, our first and foremost objective was thus to study the impact of the microcarriers and the biological glues. We therefore chose to introduce a minimum of additional factors, such as chondrogenic growth factors or mechanical stimuli, to avoid masking those effects. The results show that adult human chondrocytes were maintained and encouraged to produce ECM proteins in this system during extensive culture periods, and that the gelatin microcarriers proved essential for this outcome. Yet, lacking additional chondrogenic growth factors or physical stimulation to accelerate ECM synthesis, both in vitro models proved incapable of supporting solid cartilage formation and will need to be modified accordingly.

The gelatin microcarriers supported adhesion and expansion of human articular chondrocytes from different passages. Microcarriers made of other biomaterials have also been used for tissue engineering of cartilage, such as dextran, polystyrene, collagen, PLA, PLGA [Boyan et al., 1996, Chun et al., 2004, Hong et al., 2005, Malda and Frondoza, 2006, Chung et al., 2008, Kang et al., 2009]. Gelatin microcarriers, similar to those used in this system, have been studied with chondrocytes and chondroprogenitor cells previously [Malda et al., 2003a, Melero-Martin et al., 
2006, Huss et al., 2007]. Results showed that human nasal chondrocytes improved their re-differentiation capacity when expanded on CultiSpher-G microcarriers compared to monolayer-expanded chondrocytes [Malda et al., 2003a]. This is an important trait, as human chondrocytes have been reported to lose their ability to produce cartilage-specific macromolecules in monolayer culture [Holtzer et al., 1960, von der Mark et al., 1977]. Furthermore, recent findings suggest that cells can migrate between confluent and empty microcarriers, thereby allowing sub-cultivation by inoculation and eliminating the need for digestive enzyme treatment [Melero-Martin et al., 2006]. These traits, together allowing chondrocytes to be expanded, transported and transplanted on one matrix, motivated the choice of the related CultiSpher-GL microcarrier.

Four different biological glues were initially tested as binding agents for the cell-seeded microcarriers, one commercially available fibrin glue (Tisseel $\left.{ }^{\circledR}\right)$ in diluted form and three blood-derived glues: citrated platelet poor plasma, platelet rich plasma, and whole blood. All four were shown to fix the microcarriers in a three-dimensional shape until the matrix produced by the transplanted cells could take over this function. Positive staining for sulphated proteoglycans and aggrecan, indicating an active cartilage proteoglycan metabolism by the chondrocytes, was found in all groups. However, in this in vitro system, and with the present analysis methods, no difference between the biological glues, concerning cell density or capsule thickness, could be verified. Considering the diversity of the biological glues this is somewhat surprising, especially taking into account recent findings implying the capacity of platelet rich plasma to support chondrogenesis in a number of systems. Positive stimulatory effects on chondrocyte proliferation and matrix formation by platelet rich plasma supernatant as 
cell culture media additive have been reported previously [Akeda et al., 2006]. Other studies, examining the effect of platelet rich plasma on cell proliferation and phenotype expression in monolayer as well as in three-dimensional culture systems, also reported a proliferative effect of the plasma though with a negative effect on the chondrogenic phenotype [Gaissmaier et al., 2005, Drengk et al., 2008]. In contrast, when combined with a solid collagen matrix in vitro, fibrin glue and blood plasma enhanced the levels of cartilage specific genes [Malicev et al., 2007], and chondrocytes embedded in platelet rich plasma formed neocartilage in a subcutaneous rabbit model [Wu et al., 2007]. This beneficial effect may be contributed to the release of growth factors from platelet alpha granules upon activation, as a number of these growth factors are known chondrogenic stimulants, such as for example TGF- $ß$ and IGF and [Yoo et al., 1998, Landesberg et al., 2000, Jakob et al., 2001, Blunk et al., 2002, Weibrich et al., 2002, Gaissmaier et al., 2005, Akeda et al., 2006]. This instant burst of growth factors may however have been too short-lived to have an effect on the end result following the long-term three-dimensional in vitro culture investigated here [McCarrel and Fortier, 2009], where the citrated plasma was evaluated in a glue capacity. The use of platelet rich plasma supernatant as additive in the cell culture medium presents an alternative to ensure a continuous supply of these signals in vitro [Gaissmaier et al., 2005, Akeda et al., 2006]. Despite the lack of differences between the tested biological glues following the initial evaluation, the reported chondrogenic potential of platelet rich plasma described above, in addition with the potentially autologous origin, made this biological glue an attractive choice for future studies and subsequent experiments were thus focused on this glue.

In total, five additional experiments were conducted using platelet rich plasma alone as 
biological glue to supplement the data regarding the potential of platelet rich plasma to encapsulate pre-seeded gelatin microcarriers in vitro. All engineered constructs containing cell-seeded microcarriers remained intact and were solid enough to allow convenient handling once removed. Importantly, it was not possible to achieve this characteristic when the microcarriers were excluded. Instead, these constructs dissolved when the initial cell densities and dimensions were matched to the microcarriercontaining samples. In a recent study, primary bovine chondrocytes were used to evaluate cartilage formation in long-term stable fibrin glues, that demonstrated a critical dependency on fibrinogen concentration [Eyrich et al., 2007]. In the present work, we demonstrate that normal blood plasma glues in combination with pre-seeded microcarriers are in fact sufficiently stable to support three-dimensional cultures with human chondrocytes. This potentially autologous approach may facilitate clinical use of cultured autologous chondrocytes.

In all microcarrier groups, from all experiments and both in vitro models, we observed that cells as well as newly formed ECM were concentrated in a capsule surrounding the samples already after 4 weeks (Fig. 3 a). The increase in capsule thickness as well as the amount of cell nuclei with time suggests an ongoing cell proliferation, as well as an active ECM synthesis of these cells throughout the culture period (Fig. 3 a-c). Aggrecan is the major proteoglycan in cartilage and the positive staining for aggrecan that was found in the ECM indicates that the chondrocytes were capable of producing cartilagecharacteristic proteoglycans. However, the presence of collagen type I, a fibrocartilage marker, in combination with the absence of collagen type II reveals the immaturity of the formed ECM in this study. The chondrocytes did conversely stain positive for SOX9 and S-100, two markers used for chondrocytic phenotype. S-100 is a calcium-binding 
protein that has been suggested and used as an identifying marker for the chondrocytic phenotype during articular cartilage repair previously [Wolff et al., 1992, Zheng et al., 2007]. SOX-9 is a transcription factor that has been highly associated with the chondrocytic differentiation/chondrogenesis [Wehrli et al., 2003]. The positive correlation between SOX-9 and Collagen type II synthesis has however been challenged which may present one explanation for the lack of corresponding collagen type II staining in this study [Aigner et al., 2003].

As was mentioned previously, the majority of cells were detected at the sample surfaces from the early time points (Fig. 3 a). Whether the corresponding decrease in cellularity at the central parts of the samples was a result of cell migration or cell death at the centre, or a combination of both, is unclear. The MTT viability assay revealed viable cells on the individual microcarriers prior to biological glue encapsulation (Fig. 1 a). The cellularity throughout the macroporous gelatin microcarriers was not confirmed at this point, and as recent studies have indicated that the pores in similar microcarriers are in fact not completely interconnected a fully homogenous cellularity can not be ensured [Borg et al., 2009]. The presence of viable chondrocytes on the microcarriers should however ensure, although not homogenous, at least a consistent cell distribution within the microcarrier-/plasma constructs at the time of encapsulation. In order to maintain this cellularity and thereby enable cartilage formation throughout the entire sample, the mass transport to and from the cells residing in the centre of the samples must be resolved. This can for example be achieved by mechanically compressing the samples. Physical stimulation, such as intermittent pressure, is known to stimulate ECM production [Sah et al., 1989, Mauck et al., 2000, Waldman et al., 2004], in part by facilitation of nutrient and oxygen transport to cells within the sample. In addition, the 
use of bioreactors and different mechanical stimulation can dramatically influence the metabolic expression of chondrocytes, as was recently reviewed by Schulz et al [Schulz and Bader, 2007]. Application of mechanical stimulation will be addressed in forthcoming studies.

The gelatin microcarriers used in this study are macroporous with an average pore diameter of $30 \mu \mathrm{m}$. Previous studies have reported that the average pore diameters in gelatin scaffolds can influence the glycosaminoglycan production of rat chondrocytes [Lien et al., 2008], and both rabbit and bovine chondrocytes yielded higher contents of collagen and glycosaminoglycan content when grown on porous PLGA scaffolds compared to nonporous scaffolds [Chung et al., 2008, Kang et al., 2009]. The importance of the microcarrier porosity in this study is unclear, but in a recent study we have investigated the impact of pore diameters on human chondrocyte proliferation (Pettersson et al, submitted). This type of gelatin microcarriers is cross-linked with a non-zero length diisocyanate cross-linking agent, through the reaction of free amine groups of lysine and hydroxylysine or free carboxylic acid residues of glutamic and aspartic acid of the protein molecules [Kuijpers et al., 2000]. Concerning in vitro degradation, Kong et al has reported continuous material release from this type of gelatin microcarrier in an in vitro system with a $\mathrm{CHO}$ cell line [Kong et al., 1999]. Interestingly, in our study it was only in experiments where uniquely microcarrierexpanded cells were used (Experiment V and VI), that the microcarriers displayed clear signs of degradation (Fig. 3 h-i).

We have observed a gradual improvement of the results, with regards to capsule thickness, cell density and matrix formation, during the course of this study (Fig. 4). 
Several factors may have contributed to this trend. As illustrated by Table 1, minor adjustments have been made to the protocol that may have contributed to the positive development, most noticeably the use of exclusively microcarrier-expanded chondrocytes, but it is also important to consider possible donor-to-donor variations when interpreting the results. Regardless of this positive trend, all of the included experiments support the main hypothesis. Human articular chondrocytes from four individual donors following total knee arthroplasties, seeded on gelatin microcarriers sealed with platelet rich plasma, remained viable and retained part of their phenotype under static conditions in the absence of additional chondrogenic growth factors for extensive culture periods in vitro. Stable structures, staining positive for sulphated proteoglycans, aggrecan and two chondrocytic phenotype markers formed at similar time points for all these experiments, in pellets as well as in type I collagen coated and non-coated cell culture inserts, with minimal basal media as well as chondrocyte growth media and with monolayer-expanded as well as exclusively microcarrier-expanded chondrocytes. These results imply that microcarriers sealed with blood-derived glues is indeed a plausible delivery system for human chondrocytes.

In conclusion, we have shown that cell-seeded biodegradable porous microcarriers that are fixed with blood-derived, biodegradable glues form a stable scaffold that support ECM formation by human articular chondrocytes. The in vitro models used in this system will need to be modified; either by applying mechanical stimulation or by adding chondrogenic growth factors to the cell culture media, in order to stimulate matrix production and cartilage formation further.

\section{ACKNOWLEDGEMENTS}

This study was funded by the Swedish Foundation for Strategic Research (Grant 
Number: A3 02:139) and the Materials in Medicine strategic research area (Linköping

University and Landstinget i Östergötland).

\section{REFERENCES}

Aigner T, Gebhard PM, Schmid E, et al. 2003; SOX9 expression does not correlate with type II collagen expression in adult articular chondrocytes. Matrix Biol 22(4): 363-372.

Akeda K, An HS, Okuma M, et al. 2006; Platelet-rich plasma stimulates porcine articular chondrocyte proliferation and matrix biosynthesis. Osteoarthritis Cartilage 14(12): 1272-1280.

Blunk T, Sieminski AL, Gooch KJ, et al. 2002; Differential effects of growth factors on tissue-engineered cartilage. Tissue Eng 8(1): 73-84.

Borg DJ, Dawson RA, Leavesley DI, et al. 2009; Functional and phenotypic characterization of human keratinocytes expanded in microcarrier culture. $J$ Biomed Mater Res A 88(1): 184-194.

Boyan BD, Hummert TW, Dean DD, et al. 1996; Role of material surfaces in regulating bone and cartilage cell response. Biomaterials 17(2): 137-146. Brittberg M, Sjögren-Jansson E, Lindahl A, et al. 1997; Influence of fibrin sealant (Tisseel) on osteochondral defect repair in the rabbit knee. Biomaterials 18(3): 235-242.

Chun KW, Yoo HS, Yoon JJ, et al. 2004; Biodegradable PLGA microcarriers for injectable delivery of chondrocytes: effect of surface modification on cell attachment and function. Biotechnol Prog 20(6): 1797-1801.

Chung HJ, Kim IK, Kim TG, et al. 2008; Highly open porous biodegradable microcarriers: in vitro cultivation of chondrocytes for injectable delivery. Tissue Eng Part A 14(5): 607-615.

Demetriou AA, Reisner A, Sanchez J, et al. 1988; Transplantation of microcarrier-attached hepatocytes into $90 \%$ partially hepatectomized rats. Hepatology 8(5): 1006-1009.

Drengk A, Zapf A, Sturmer EK, et al. 2008; Influence of Platelet-Rich Plasma on Chondrogenic Differentiation and Proliferation of Chondrocytes and Mesenchymal Stem Cells. Cells Tissues Organs

Eyrich D, Brandl F, Appel B, et al. 2007; Long-term stable fibrin gels for cartilage engineering. Biomaterials 28(1): 55-65.

Fernandes AM, Fernandes TG, Diogo MM, et al. 2007; Mouse embryonic stem cell expansion in a microcarrier-based stirred culture system. J Biotechnol 132(2): 227-236.

Frondoza C, Sohrabi A, Hungerford D. 1996; Human chondrocytes proliferate and produce matrix components in microcarrier suspension culture.

Biomaterials 17(9): 879-888.

Gaissmaier C, Fritz J, Krackhardt T, et al. 2005; Effect of human platelet supernatant on proliferation and matrix synthesis of human articular chondrocytes in monolayer and three-dimensional alginate cultures.

Biomaterials 26(14): 1953-1960.

Goessl A, Redl H. 2005; Optimized thrombin dilution protocol for a slowly setting fibrin sealant in surgery. Eur Surg 37(1): 43-51.

Holtzer H, Abbott J, Lash J, et al. 1960; The Loss of Phenotypic Traits by 
Differentiated Cells in Vitro, I. Dedifferentiation of Cartilage Cells. Proc Natl Acad Sci U S A 46(12): 1533-1542.

Homminga GN, Buma P, Koot HW, et al. 1993; Chondrocyte behavior in fibrin glue in vitro. Acta Orthop Scand 64(4): 441-445.

Hong Y, Gao C, Xie Y, et al. 2005; Collagen-coated polylactide microspheres as chondrocyte microcarriers. Biomaterials 26(32): 6305-6313.

Huss FR, Junker JP, Johnson H, et al. 2007; Macroporous gelatine spheres as culture substrate, transplantation vehicle, and biodegradable scaffold for guided regeneration of soft tissues. In vivo study in nude mice. J Plast Reconstr Aesthet Surg 60(5): 543-555.

Jakob M, Demarteau O, Schafer D, et al. 2001; Specific growth factors during the expansion and redifferentiation of adult human articular chondrocytes enhance chondrogenesis and cartilaginous tissue formation in vitro. $J$ Cell Biochem 81(2): 368-377.

Kang SW, La WG, Kim BS. 2009; Open Macroporous Poly(lactic-co-glycolic Acid) Microspheres as an Injectable Scaffold for Cartilage Tissue Engineering. $J$ Biomater Sci Polym Ed 20(3): 399-409.

Kong D, Chen M, Gentz R, et al. 1999; Cell growth and protein formation on various microcarriers. Cytotechnology 29(2): 151-158.

Kuijpers AJ, Engbers GH, Krijgsveld J, et al. 2000; Cross-linking and characterisation of gelatin matrices for biomedical applications. J Biomater Sci Polym Ed 11(3): 225-243.

Landesberg R, Roy M, Glickman RS. 2000; Quantification of growth factor levels using a simplified method of platelet-rich plasma gel preparation. J Oral Maxillofac Surg 58(3): 297-300; discussion 300-291.

Lien SM, Ko LY, Huang TJ. 2008; Effect of pore size on ECM secretion and cell growth in gelatin scaffold for articular cartilage tissue engineering. Acta Biomater

Liu JY, Hafner J, Dragieva G, et al. 2004; Autologous cultured keratinocytes on porcine gelatin microbeads effectively heal chronic venous leg ulcers. Wound Repair Regen 12(2): 148-156.

Malda J, Frondoza CG. 2006; Microcarriers in the engineering of cartilage and bone. Trends Biotechnol 24(7): 299-304.

Malda J, Kreijveld E, Temenoff JS, et al. 2003a; Expansion of human nasal chondrocytes on macroporous microcarriers enhances redifferentiation.

Biomaterials 24(28): 5153-5161.

Malda J, van Blitterswijk CA, Grojec M, et al. 2003b; Expansion of bovine chondrocytes on microcarriers enhances redifferentiation. Tissue Eng 9(5): 939948.

Malicev E, Radosavljevic D, Velikonja NK. 2007; Fibrin gel improved the spatial uniformity and phenotype of human chondrocytes seeded on collagen scaffolds. Biotechnol Bioeng 96(2): 364-370.

Mauck RL, Soltz MA, Wang CC, et al. 2000; Functional tissue engineering of articular cartilage through dynamic loading of chondrocyte-seeded agarose gels. J Biomech Eng 122(3): 252-260.

McCarrel T, Fortier L. 2009; Temporal growth factor release from platelet-rich plasma, trehalose lyophilized platelets, and bone marrow aspirate and their effect on tendon and ligament gene expression. J Orthop Res

Meinhart J, Fussenegger M, Hobling W. 1999; Stabilization of fibrin-chondrocyte constructs for cartilage reconstruction. Ann Plast Surg 42(6): 673-678. 
Melero-Martin JM, Dowling MA, Smith M, et al. 2006; Expansion of chondroprogenitor cells on macroporous microcarriers as an alternative to conventional monolayer systems. Biomaterials 27(15): 2970-2979.

Mercier NR, Costantino HR, Tracy MA, et al. 2005; Poly(lactide-co-glycolide) microspheres as a moldable scaffold for cartilage tissue engineering.

Biomaterials 26(14): 1945-1952.

Ohlson S, Branscomb J, Nilsson K. 1994; Bead-to-bead transfer of Chinese hamster ovary cells using macroporous microcarriers. Cytotechnology 14(1): 67-80.

Overstreet M, Sohrabi A, Polotsky A, et al. 2003; Collagen microcarrier spinner culture promotes osteoblast proliferation and synthesis of matrix proteins. In Vitro Cell Dev Biol Anim 39(5-6): 228-234.

Sah RL, Kim YJ, Doong JY, et al. 1989; Biosynthetic response of cartilage explants to dynamic compression. J Orthop Res 7(5): 619-636.

Schulz RM, Bader A. 2007; Cartilage tissue engineering and bioreactor systems for the cultivation and stimulation of chondrocytes. Eur Biophys J 36(4-5): 539568.

Silverman RP, Passaretti D, Huang W, et al. 1999; Injectable tissue-engineered cartilage using a fibrin glue polymer. Plast Reconstr Surg 103(7): 1809-1818. van Wezel AL. 1967; Growth of cell-strains and primary cells on micro-carriers in homogeneous culture. Nature 216(5110): 64-65.

Voigt M, Schauer M, Schaefer DJ, et al. 1999; Cultured epidermal keratinocytes on a microspherical transport system are feasible to reconstitute the epidermis in full-thickness wounds. Tissue Eng 5(6): 563-572.

von der Mark K, Gauss V, von der Mark H, et al. 1977; Relationship between cell shape and type of collagen synthesised as chondrocytes lose their cartilage phenotype in culture. Nature 267(5611): 531-532.

Waldman SD, Spiteri CG, Grynpas MD, et al. 2004; Long-term intermittent compressive stimulation improves the composition and mechanical properties of tissue-engineered cartilage. Tissue Eng 10(9-10): 1323-1331.

Wehrli BM, Huang W, De Crombrugghe B, et al. 2003; Sox9, a master regulator of chondrogenesis, distinguishes mesenchymal chondrosarcoma from other small blue round cell tumors. Hum Pathol 34(3): 263-269.

Weibrich G, Kleis WK, Hafner G, et al. 2002; Growth factor levels in platelet-rich plasma and correlations with donor age, sex, and platelet count. $J$

Craniomaxillofac Surg 30(2): 97-102.

Werner A, Duvar S, Muthing J, et al. 2000; Cultivation of immortalized human hepatocytes HepZ on macroporous CultiSpher G microcarriers. Biotechnol Bioeng 68(1): 59-70.

Wolff DA, Stevenson S, Goldberg VM. 1992; S-100 protein immunostaining identifies cells expressing a chondrocytic phenotype during articular cartilage repair. J Orthop Res 10(1): 49-57.

Wu W, Chen F, Liu Y, et al. 2007; Autologous injectable tissue-engineered cartilage by using platelet-rich plasma: experimental study in a rabbit model. $J$ Oral Maxillofac Surg 65(10): 1951-1957.

Yoo JU, Barthel TS, Nishimura K, et al. 1998; The chondrogenic potential of human bone-marrow-derived mesenchymal progenitor cells. J Bone Joint Surg Am 80(12): 1745-1757.

Young S, Wong M, Tabata Y, et al. 2005; Gelatin as a delivery vehicle for the controlled release of bioactive molecules. J Control Release 109(1-3): 256-274. 
Zheng MH, Willers C, Kirilak L, et al. 2007; Matrix-induced autologous chondrocyte implantation (MACl): biological and histological assessment. Tissue Eng 13(4): 737-746. 


\section{TABLES}

Table 1. List of the included experiments and protocol adjustments. Passage refers to the cell passage number at microcarrier seeding ( $\mathrm{P} 0$ refers to exclusively microcarrierexpanded cells). For the in vitro models, COLL-I refers to type I collagen coated cell culture insert membranes and dimension refers to the initial clot dimensions (insert) or the amount of microcarriers and biological glue (pellet). Time points refer to the number of hours $(\mathrm{h})$, days $(\mathrm{d})$ or weeks $(\mathrm{w})$ of culture that preceded sampling. $\mathrm{BM}=$ basal medium. $\mathrm{CM}=$ chondrocyte growth medium.

\begin{tabular}{ccccccc}
\hline Exp & Donor & Passage & Model & Dimension & Time points & Medium \\
\hline I & A & P3 & Insert COLL-I & $2 m m(h) \times 6.4 m m(\varnothing)$ & $4,8,12,16 \mathrm{w}$ & BM \\
II & B & P4 & Insert COLL-I & $4 \mathrm{~mm}(\mathrm{~h}) \times 10.5 \mathrm{~mm}(\varnothing)$ & $4,8,12,17 \mathrm{w}$ & BM \\
III & C & P2 & Pellet & $8 \mathrm{mg}+150 \mu \mathrm{l}$ & $2,6,12 \mathrm{w}$ & CM \\
IV & C & P3 & Pellet & $8 \mathrm{mg}+150 \mu \mathrm{l}$ & $4 \mathrm{~h}, 2 \mathrm{~d}, 7 \mathrm{~d}$ & CM \\
V & D & P0 & Insert & $4 \mathrm{~mm}(\mathrm{~h}) \times 10.5 \mathrm{~mm}(\varnothing)$ & $4,8,12 \mathrm{w}$ & CM \\
VI & D & P0 & Pellet & $8 \mathrm{mg}+100 \mu \mathrm{l}$ & $4,8,12 \mathrm{w}$ & CM \\
\hline
\end{tabular}



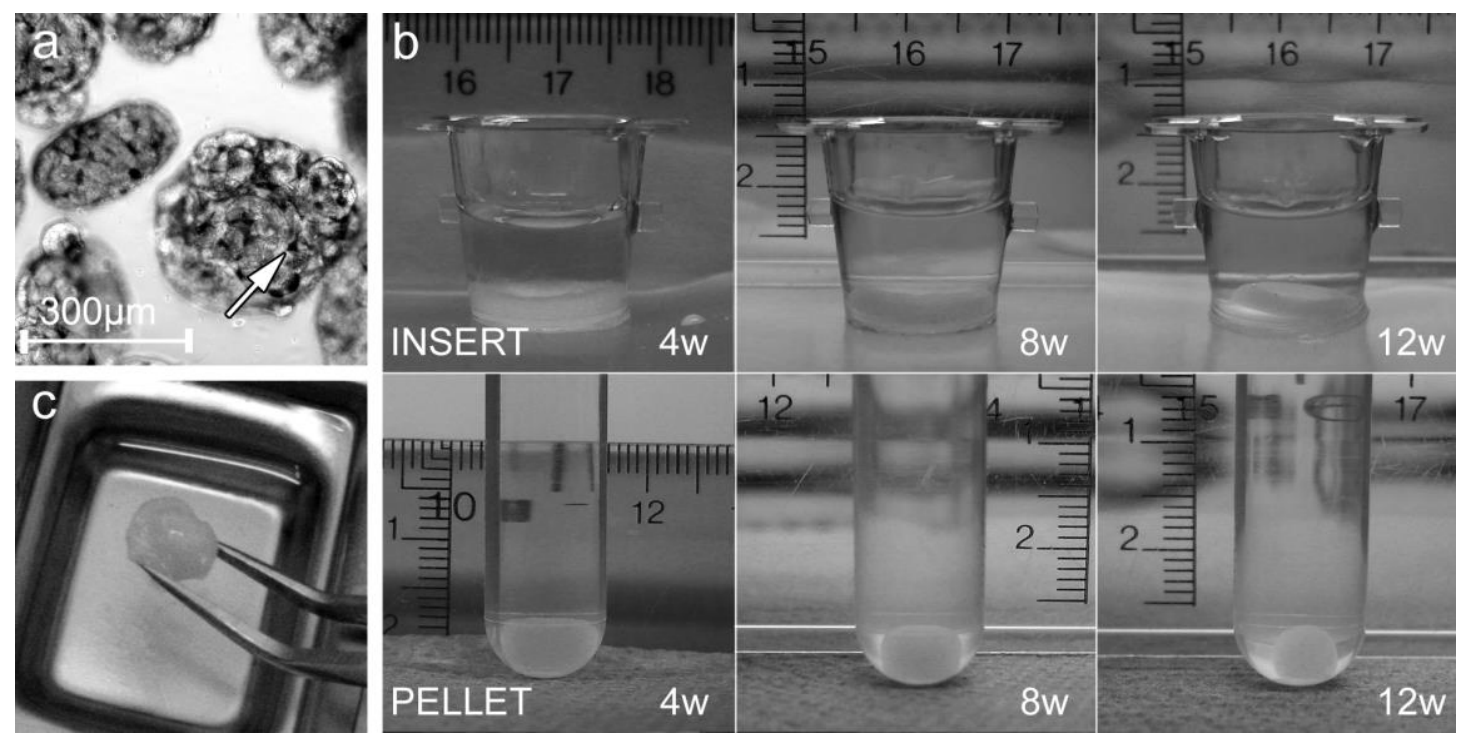

Fig. 1. a) Inverted light microscopy image of MTT-stained human chondrocytes on CultiSpher-GL microcarriers two days after initial cell seeding. The arrow indicates MTT-formazan reaction in the cytoplasm of viable chondrocytes. Cells are homogeneously distributed among the microcarriers as well as on each microcarrier. Experiment I. b) Images of the two in vitro models used during this study. Chondrocyte-seeded microcarriers were encapsulated in either cell culture inserts (top row) or in polypropylene tubes for pellet culture (bottom row). Images were taken after 4 (left), 8 (center) and 12 (right) weeks of culture respectively. The clots detached from the cell culture inserts and all clots contracted during the culture period, as can be seen by the decrease in clot size with time. Experiments V and VI. c) Cartilage sample, containing pre-seeded microcarriers, photographed after completed dehydration following 17 weeks of culture. The samples allowed for handling from the first time point. Experiment II. 


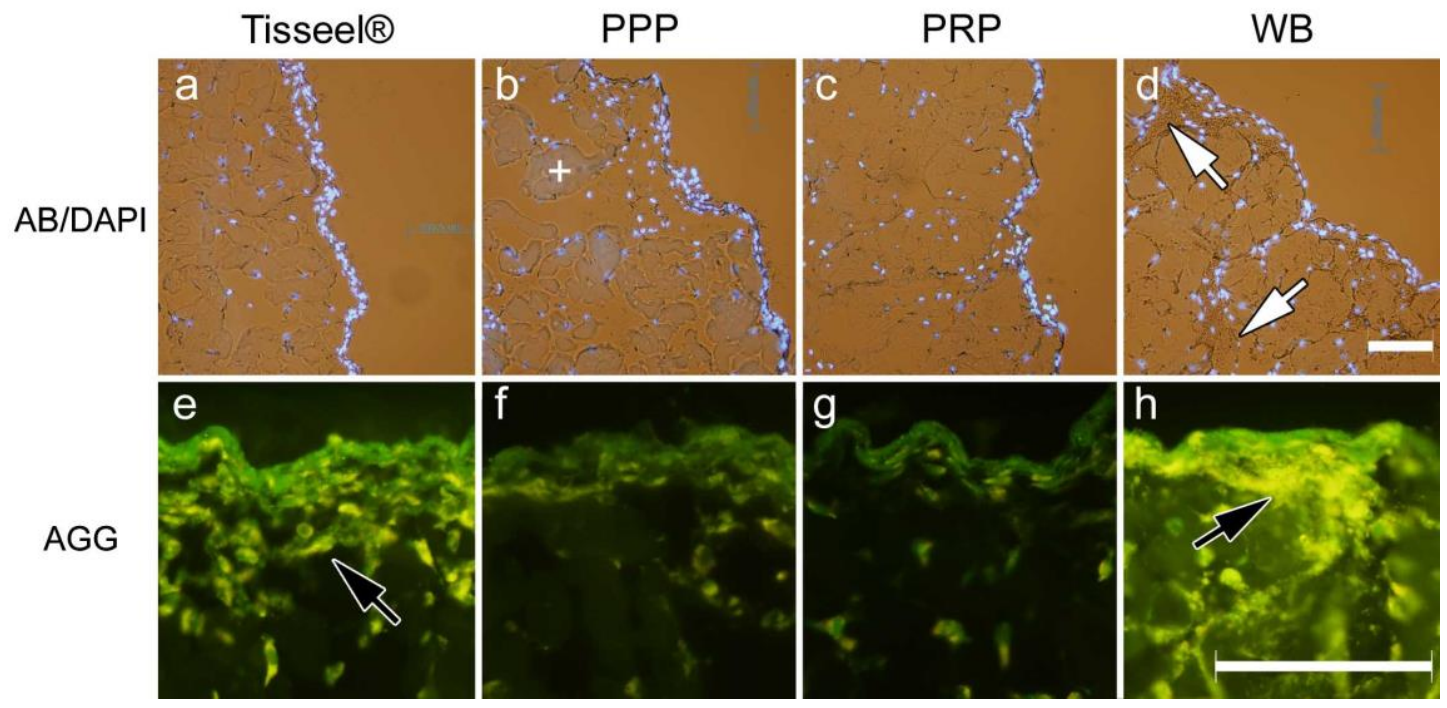

Fig. 2. Microscopy images, using a mix of white and ultraviolet light, of alcian blue and DAPI stained sections from the initial evaluation of biologal glues (a-d). Fluorescent cell nuclei can be seen primarily at the sample perimeters, with modest ECM formation below sample surfaces. The plus sign indicates a gelatin microcarrier and the white arrow indicates remnants of the biological glue for group D (whole blood), where these remnants were most evident. Positive staining for aggrecan can be seen in the peripheral ECM (e-h). Black arrows indicate non-specific yellow fluorescence. Pre-seeded microcarriers were encapsulated in Tisseel $®(a, e)$, platelet poor plasma (b, f), platelet rich plasma (c, g) and whole blood $(\mathrm{d}, \mathrm{h})$, and cultured in vitro for 16 weeks prior to sampling. Scale bars $100 \mu \mathrm{m}$. Experiment I. 


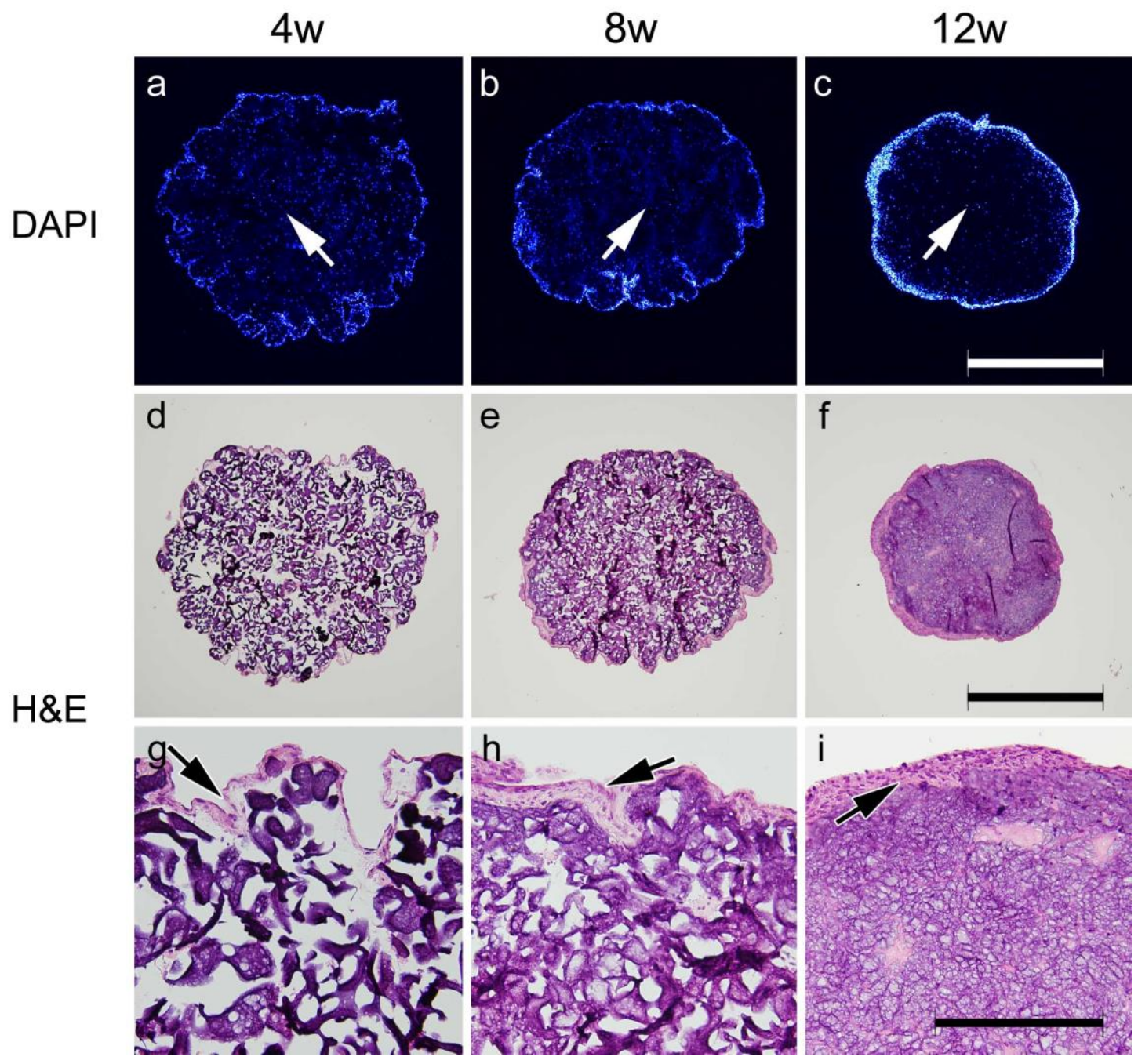

Fig. 3. Images of cartilage pellet samples stained with DAPI (a-c) and H\&E (d-i), following 4 (a, d, g), 8 (b, e, h) and 12 (c, f, i) weeks of culture respectively. The cell density is concentrated to the superficial parts of the pellets, but fluorescent cell nuclei can be found in the central parts at all time points (white arrows). The amount of cells and ECM increased with time at the circumference (left to right), resulting in progressively thicker capsules (black arrows). After 8 weeks the microcarriers had started to degrade (h) and the degradation was more prominent after 12 weeks (i). Images in two top rows are at the same magnification (scale bars $1.25 \mathrm{~mm}$ ). Images in bottom row are at the same magnification (scale bar $250 \mu \mathrm{m}$ ). Experiment VI. 

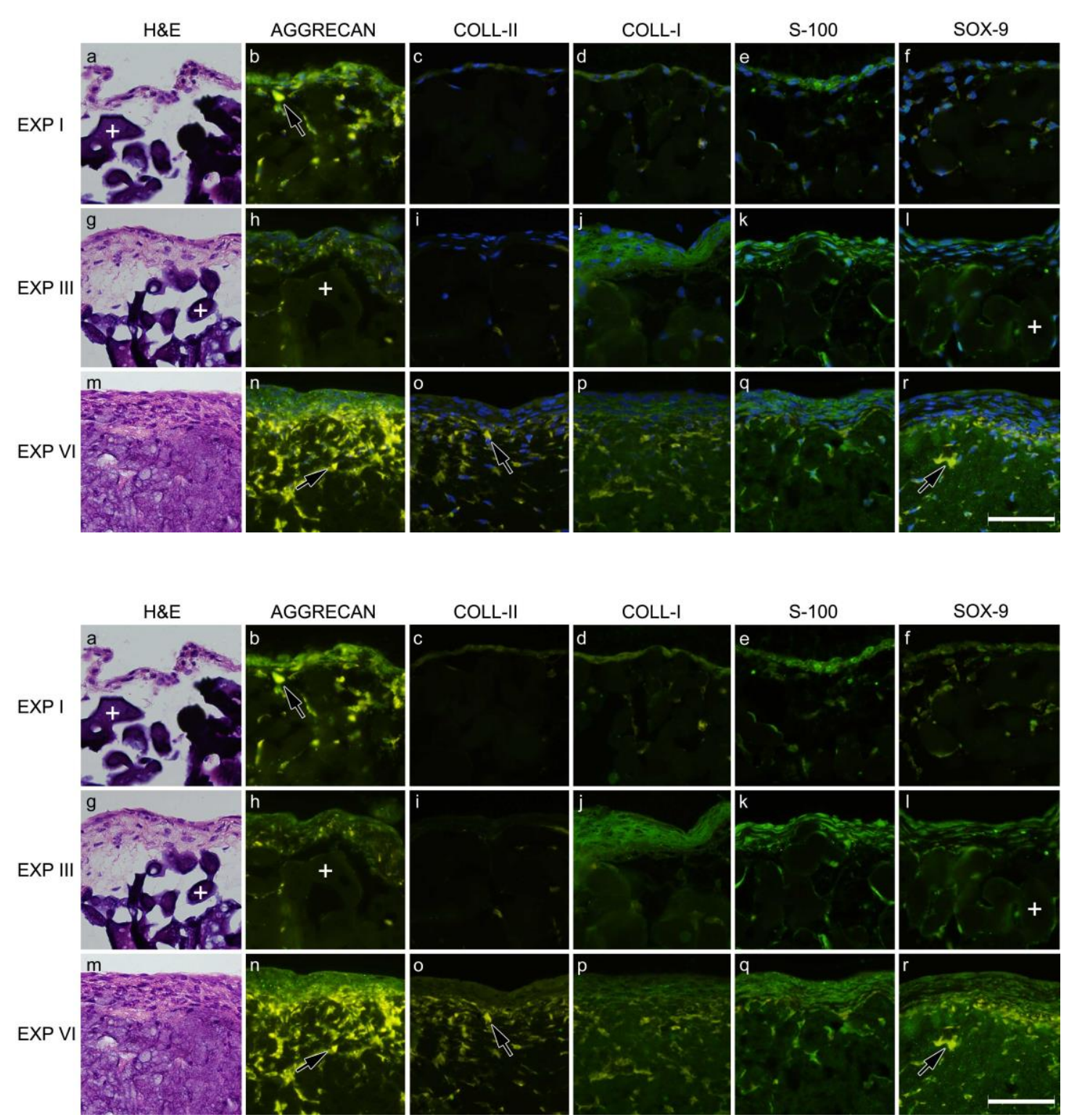

Fig. 4. Stained sections from three long-term experiments (I, III and VI), showing sample surfaces following 12 weeks of culture. A gradual increase in capsule thickness and cell density can be seen between the experiments (top to bottom row). For experiment VI, using exclusively microcarrier-expanded chondrocytes, the microcarriers had started to degrade at this time (m). Indirect immunohistochemistry confirmed presence of aggrecan $(b, h, n)$ and collagen type I $(d, j, p)$ but not collagen type II (c, I, o). Chondrocytes stained positive for S-100 (e, k, q) and SOX-9 (f, 1, r) in all experiments. Cell nuclei are counterstained blue with DAPI for immunohistochemical analysis images. Gelatin microcarriers are indicated by plus 
signs. Some non-specific yellow fluorescence (black arrows) is also present. Comparable images, i.e for each antibody, were retrieved under identical conditions. All images are at the same magnification. Scale bar $50 \mu \mathrm{m}$. Experiments I, III and VI. 
ABBREVIATIONS

ECM extracellular matrix

DMEM Dulbecco's modified Eagle's medium

FCS fetal calf serum

EDTA ethylene diamine tetra-acetic acid

NCS newborn calf serum

PBS phosphate buffered saline

MTT 3-(4, 5-dimethylthiazol-2-yl)-2, 5-diphenyltetrazolium bromide

DAPI 4'-6-Diamidino-2-phenylindole

BSA bovine serum albumin 